\title{
Dietary pulp from Fructus Schisandra Chinensis supplementation reduces serum/hepatic lipid and hepatic glucose levels in mice fed a normal or high cholesterol/bile salt diet
}

\author{
Nan Sun ${ }^{1}$, Si-Yuan Pan ${ }^{1 *}$, Yi Zhang ${ }^{1}$, Xiao-Yan Wang ${ }^{1}$, Pei-Li Zhu' ${ }^{1}$ Zhu-Sheng Chu', Zhi-Ling Yu²*, \\ Shu-Feng Zhou ${ }^{3}$ and Kam-Ming Ko ${ }^{4}$
}

\begin{abstract}
Background: Recently, it has been found that Fructus Schisandra Chinensis (FSC), a Chinese herbal medicine, and its related compounds have a profound impact on lipid metabolism process. FSC can be divided into two parts, i.e., seed and pulp. The current study aimed to examine the effect of aqueous extracts of FSC pulp (AqFSC-P) on serum/hepatic lipid and glucose levels in mice fed with a normal diet (ND) or a high cholesterol/bile salt diet (HCBD).

Methods: The AqFSC-P used in the present study was fractionated into supernatant (SAgFSC-P) and precipitate (PAqFSC-P) separated by centrifugation. Male ICR mice were fed with ND or HCBD, without or with supplementation of $1 \%, 3 \%$, or $9 \%(\mathrm{w} / \mathrm{W}$ ) SAgFSC-P or PAqFSC-P for 10 days. Biomarkers were determined according to the manufacturer's instruction.

Results: Supplementation with SAgFSC-P or PAqFSC-P significantly reduced serum and hepatic triglyceride levels (approximately 40\%) in ND- and/or HCBD-fed mice. The supplementation with SAqFSC-P or PAqFSC-P reduced hepatic total cholesterol levels (by $27-46 \%$ ) in HCBD-fed mice. Supplementation with SAgFSC-P or PAqFSC-P markedly lowered hepatic glucose levels (by $13-30 \%$ ) in ND- and HCBD-fed mice. SAqFSC-P decreased serum alanine aminotransferase (ALT) activity, but PAqFSC-P increased hepatic protein contents in ND-fed mice. Bicylol, as a positive control, reduced ALT activity. In addition, mice supplemented with FSC-P or bicylol showed a smaller body weight gain and adipose tissue mass as compared to the respective un-supplemented ND- or HCBD-fed mice.

Conclusion: The results indicate that SAqFSC-P and PAqFSC-P produce hepatic lipid- and glucose-lowering as well as serum TG-lowering effects in hypercholesterolemic mice. FSC pulp may provide a safe alternative for the management of fatty liver and/or lipid disorders in humans.
\end{abstract}

Keywords: Fructus Schisandrae Chinensis, Bicyclol, Fatty liver, Hypercholesterolemia, Epididymal fat, Glucose

\section{Background}

A growing body of evidence has shown that hyperlipidemia, regardless of cause, is an important factor involved in the development of a wide range of human diseases, including coronary heart disease (CHD) [1,2], nonalcoholic fatty liver disease (NAFLD) [3] and metabolic syndrome

\footnotetext{
* Correspondence: siyuanpan@163.com; yuzhiling@hotmail.com

'Department of Pharmacology, School of Chinese Materia Medica, Beijing University of Chinese Medicine, Beijing 100102, China

${ }^{2}$ School of Chinese Medicine, Hong Kong Baptist University, Hong Kong, China
} Full list of author information is available at the end of the article
[4]. Therefore, the searching for therapeutic intervention aimed at lowering lipid levels in blood and the liver has been an area of intensive research. While lipid-lowering drugs can be used to lower the elevated levels of lipids in patients with dyslipidemia [5], there is still no effective drug for the treatment of NAFLD, which is manifested as deposition of fat in the liver in patients without an excessive consumption of alcohol. The commonly prescribed lipid-lowering drugs can cause many potential adverse/side effects, such as myopathies, renal impairment, hepatic injury, and or pancreatitis [6,7]. Therefore, it is of clinical
C Biomed Central 
interest to manage hyperlipidemia with naturally-occurring ingredients such as coenzyme Q10 [8], phytosterols [9], unsaturated fatty acids $[10,11]$, probiotics [12], as well as herbs $[13,14]$.

Fructus Schisandra Chinensis (FSC, Wu-wei-zi in Chinese), a traditional Chinese tonic herb, has been used for thousands of years in China. In generally, FSC can be divided into two parts - seed and pulp. Previous studies have shown that FSC extract (both seed and pulp) $[15,16]$ or its active ingredient schisandrin B [17] and other related compounds bicyclol [18] and bifendate [19] can reduce hepatic triglyceride (TG) and total cholesterol (TC) levels in mice with hypercholesterolemia induced by high-fat diet containing cholesterol/bile salt. In this study, we endeavored to investigate whether the two fractions of the aqueous extract of FSC pulp (AqFSC-P), namely, supernatant (SAqFSC-P) and precipitate (PAqFSC-P), can decrease serum and hepatic lipid/glucose (GLU) levels in normal and hypercholesterolemic mice. Serum or hepatic lipids including TG, TC, low-density lipoprotein (LDL), high-density lipoprotein (HDL), as well as HDL/ LDL, LDL/HDL, and non-HDL (N-HDL) were measured. Bicyclol, a synthetic derivative of dibenzocyclooctadiene lignans from the FSC, was used as positive control for comparison. The objective of this study is to establish the pharmacological basis for the potential use of FSC-P for the treatment of the patients with hypertriglyceridemia, fatty liver disease, and obesity. As the residue from the manufacture process of FSC is often treated as waste, the effects of supplementation with the precipitate of AqFSC$\mathrm{P}$ on lipids were compared with those of the supernatant of AqFSC-P.

\section{Results}

\section{Serum lipid levels}

Supplementation with SAqFSC-P and PAqFSC-P did not affect serum TG and TC levels in mice fed with ND, except for PAqFSC-P at a dose of $1 \%$ which showed a significant decrease in serum TG level (Figure 1). While HCBD feeding slightly decreased serum TG level, both SAqFSC-P and PAqFSC-P supplementations further decreased serum TG level (by $23-30 \%$ ) in HCBD-fed (i.e., hypercholesterolemic) mice (Figure 1A). HCBD feeding markedly increased serum TC level by $53 \%$ in mice (Figure 1B), but PAqFSC-P supplementations did not affect serum TC levels in HCBD-fed mice. SAqFSC-P (3\%) decreased serum TC levels in hypercholesterolemic mice. Bicyclol supplementation did not affect serum TG and $\mathrm{TC}$ levels in both ND- and HCBD-fed mice. In addition, although HCBD intake did not affect the serum HDL levels, it increased serum LDL level, LDL/HDL ratio, and N-HDL level by $440 \%, 500 \%$, and $375 \%$, respectively, and decreased the HDL/LDL ratio by $83 \%$. FSC-P treatment did not affect serum HDL and LDL level in ND-fed
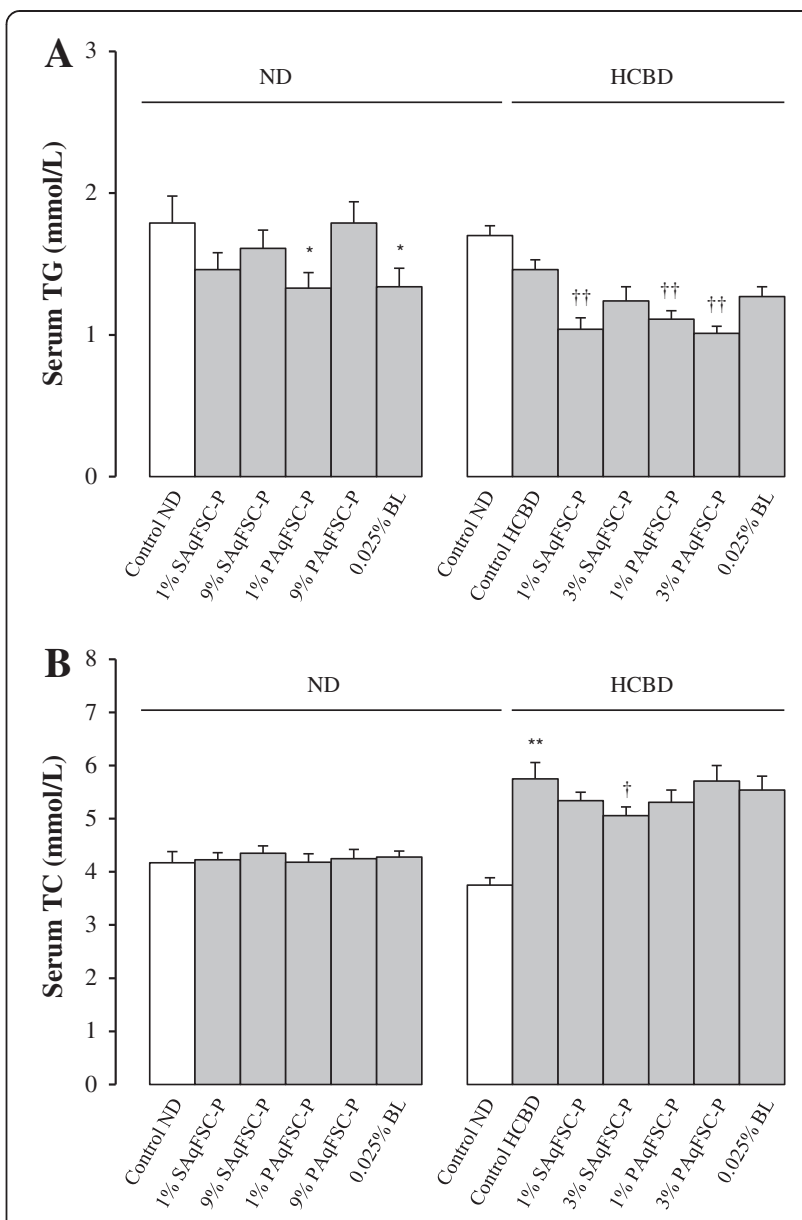

Figure 1 Effects of dietary supplementation with SAqFSC-P, PAqFSC-P, or bicyclol on serum TG and TC levels in normal and hypercholesterolemic mice. Mice were fed with normal diet (ND) or high cholesterol/bile salt $(1 \% / 0.3 \%$, w/w) diet (HCBD) without and with the supplementation with supernatant/precipitate of aqueous extract of pulp from Fructus Schisandrae Chinesis [namely, SAgFSC-P/ PAqFSC-P (1\%, 3\%, or 9\%, w/w)] or bicyclol (BL, 0.025\%, w/w) for 10 days. The concentrations of SAgFSC-P/PAgFSC-P were estimated on the basis of crude herbal material. Serum triglyceride (TG) and total cholesterol (TC) levels (as indicated in Figure $\mathbf{A}$ and $\mathbf{B}$, respectively) were measured. Value given are the means \pm S.E.M., with $n=10 .{ }^{*} P<0.05,{ }^{* *} P<0.01$ vs. control $\mathrm{ND}_{i}{ }^{\dagger} P<0.05,{ }^{+\dagger} P<0.01$ vs. control HCBD, using a one-way ANOVA followed by Dunnett's multiple comparisons test or post-hoc analysis.

mice. However, supplementation with PAqFSC-P (1\% or $3 \%$ ) attenuated N-HDL levels by 15 and $22 \%$, respectively, in HCBD-fed mice, when compared the un-supplemented HCBD-fed mice (Table 1).

\section{Hepatic lipid levels}

Mice fed ND supplemented with $1 \%$ or 9\% SAqFSC-P for 10 days showed a significant decrease in hepatic TG content by 9 and 22\%, respectively (Figure 2A). Supplementation with SAqFSC-P or PAqFSC-P at doses of $1 \%$ and $9 \%$ increased hepatic TC contents (by $6-18 \%$ ) 
Table 1 Effects of dietary supplementation with SAqFSC-P, PAqFSC-P, or bicyclol on serum lipoprotein profiles in normal and hypercholesterolemic mice

\begin{tabular}{|c|c|c|c|c|c|c|}
\hline Groups & $\begin{array}{l}\text { Drug concentration } \\
(\%, w / w)\end{array}$ & $\mathrm{HDL}(\mathrm{mmol} / \mathrm{L})$ & LDL (mmol/L) & HDL/LDL (mmol/L) & LDL/HDL (mmol/L) & $\mathrm{N}-\mathrm{HDL}(\mathrm{mmol} / \mathrm{L})$ \\
\hline \multicolumn{7}{|l|}{ Normal mice } \\
\hline Control ND & - & $3.94 \pm 0.21$ & $0.71 \pm 0.04$ & $5.82 \pm 0.34$ & $0.18 \pm 0.01$ & $0.11 \pm 0.11$ \\
\hline \multirow[t]{2}{*}{ SAqFSC-P/ND } & 1 & $3.95 \pm 0.09$ & $0.67 \pm 0.03$ & $6.02 \pm 0.28$ & $0.17 \pm 0.01$ & $0.23 \pm 0.13$ \\
\hline & 9 & $4.00 \pm 0.11$ & $0.63 \pm 0.04$ & $6.47 \pm 0.30$ & $0.16 \pm 0.01$ & $0.25 \pm 0.09$ \\
\hline \multirow[t]{2}{*}{ PAqFSC-P/ND } & 1 & $4.00 \pm 0.15$ & $0.63 \pm 0.03$ & $6.20 \pm 0.36$ & $0.16 \pm 0.01$ & $0.23 \pm 0.06$ \\
\hline & 9 & $4.18 \pm 0.17$ & $0.63 \pm 0.03$ & $6.46 \pm 0.29$ & $0.16 \pm 0.01$ & $0.17 \pm 0.06$ \\
\hline Bicyclol/ND & 0.025 & $4.17 \pm 0.16$ & $0.70 \pm 0.04$ & $6.21 \pm 0.48$ & $0.17 \pm 0.02$ & $0.10 \pm 0.07$ \\
\hline \multicolumn{7}{|c|}{ Hypercholester-olemic mice } \\
\hline Control ND & - & $3.10 \pm 0.12$ & $0.20 \pm 0.02$ & $16.65 \pm 1.30$ & $0.06 \pm 0.01$ & $0.64 \pm 0.03$ \\
\hline Control HCBD & - & $3.02 \pm 0.12$ & $1.08 \pm 0.07^{\mathrm{a}}$ & $2.86 \pm 0.17^{\mathbf{a}}$ & $0.36 \pm 0.02^{\mathbf{a}}$ & $2.54 \pm 0.14^{\mathbf{a}}$ \\
\hline \multirow[t]{2}{*}{ SAqFSC-P/HCBD } & 1 & $3.15 \pm 0.15$ & $1.08 \pm 0.06$ & $3.29 \pm 0.16$ & $0.31 \pm 0.02$ & $2.17 \pm 0.12$ \\
\hline & 3 & $3.20 \pm 0.12$ & $1.22 \pm 0.09$ & $2.90 \pm 0.14$ & $0.35 \pm 0.02$ & $2.51 \pm 0.19$ \\
\hline \multirow[t]{2}{*}{ PAqFSC-P/HCBD } & 1 & $3.16 \pm 0.10$ & $1.09 \pm 0.06$ & $2.72 \pm 0.17$ & $0.37 \pm 0.02$ & $2.18 \pm 0.10^{\mathbf{b}}$ \\
\hline & 3 & $3.08 \pm 0.10$ & $0.93 \pm 0.05$ & $3.30 \pm 0.21$ & $0.35 \pm 0.02$ & $1.98 \pm 0.11^{\mathbf{b}}$ \\
\hline Bicyclol/HCBD & 0.025 & $3.27 \pm 0.14$ & $1.02 \pm 0.07$ & $3.41 \pm 0.23$ & $0.31 \pm 0.02$ & $2.28 \pm 0.13$ \\
\hline
\end{tabular}

Experimental details were described in Figure 1. Mice were fed with ND or HCBD containing SAqFSC-P, PAqFSC-P, or bicyclol at the indicated concentration for 10 days. Serum low-density lipoprotein (LDL), high-density lipoprotein (HDL), HDL/LDL, LDL/HDL, and N-HDL levels were then measured. Mouse model of hypercholesterolemia was generated by HCBD. Values given are the means \pm S.E.M., with $\mathrm{n}=10{ }^{\mathbf{a}} P<0.01$ vs. control ND; ${ }^{\mathbf{b}} P<0.05$ vs. control HCBD, using a one-way ANOVA follow by Dunnett's multiple comparisons test or post-hoc analysis.

in ND-fed mice (Figure 2B). Feeding mice with HCBD markedly increased hepatic TG and TC contents (by 196 and $339 \%$, respectively), when compared with those of mice fed with ND. Supplementation with SAqFSC-P and PAqFSC-P at $1 \%$ or $3 \%$ decreased hepatic TC and TG contents (by $27-46 \%$ and $40-42 \%$, respectively) in HCBD-fed mice. However, 3\% PAqFSC-P did not decrease hepatic TC in hypercholesterolemic mice. While 0.025\% bicyclol supplementation reduced hepatic TG content (by 32\%) in ND-fed mice, it did not affect hepatic TG and TC contents in HCBD-fed mice.

\section{Serum and hepatic glucose levels}

While supplementation with SAqFSC-P or PAqFSC-P at a dose of $1 \%$ and $9 \%$ did not affect the serum GLU levels in ND- and HCBD-fed mice, HCBD feeding significantly decreased serum GLU level by $11 \%$ (Figure 3A). SAqFSC-P or PAqFSC-P supplementation at doses of $1 \%$ and $9 \%$ decreased hepatic GLU level (by $16-31 \%$ ) in ND-fed mice. HCBD feeding increased hepatic GLU level by $19 \%$, when compared with that of mice fed with ND. Supplementation with SAqFSC-P or PAqFSC-P at $1 \%$ and $3 \%$ decreased hepatic GLU level (by $12-18 \%$ ) in HCBD-fed mice (Figure 3B). While bicyclol supplementation produced no detectable changes in serum GLU levels in both ND- and HCBD-fed mice, it decreased hepatic GLU level (by 32\%) in ND-fed but not HCBDfed mice.

\section{Hepatic index/protein/function}

Supplementation with PAqFSC-P at 9\% increased hepatic index by $9 \%$ in ND-fed mice, but not in HCBD-fed mice. PAqFSC-P supplementation at 1 or $9 \%$ increased hepatic protein content by 6 and 12\% in ND-fed mice, respectively. However, only $1 \%$ SAqFSC-P supplementation decreased hepatic protein level by $7 \%$ in hypercholesterolemic mice, when compared with the un-supplemented HCBD-fed mice. Bicyclol treatment increased hepatic protein by $11 \%$ in ND-fed mice. SAqFSC-P and bicyclol supplementation suppressed serum ALT activity by 24 and 29\%, respectively, in ND-fed mice. Bicyclol also inhibited ALT activity by $42 \%$ in HCBD-fed mice. HCBD feeding increased hepatic weight, hepatic protein content, and ALT activity (by 12, 13 and 38\%, respectively) (Table 2).

\section{Fatty index}

Supplementation with SAqFSC-P at $1 \%$ and $9 \%$ or PAq FSC-P at $1 \%$ decreased the fatty index (by 15 and $18 \%$ or $16 \%$, respectively) in ND-fed mice. HCBD feeding did not cause any detectable change in fatty index in mice, and both SAqFSC-P and PAqFSC-P supplementation did not produce any changes in fatty index in HCBD-fed mice, except for 1\% PAqFSC-P supplementation which caused a slight but significant decrease (by 13\%) in fatty index. While bicyclol supplementation at $0.025 \%$ reduced the fatty index (by 19\%) in ND-fed mice, it did not cause any effect on HCBD-fed mice (Figure 4). 


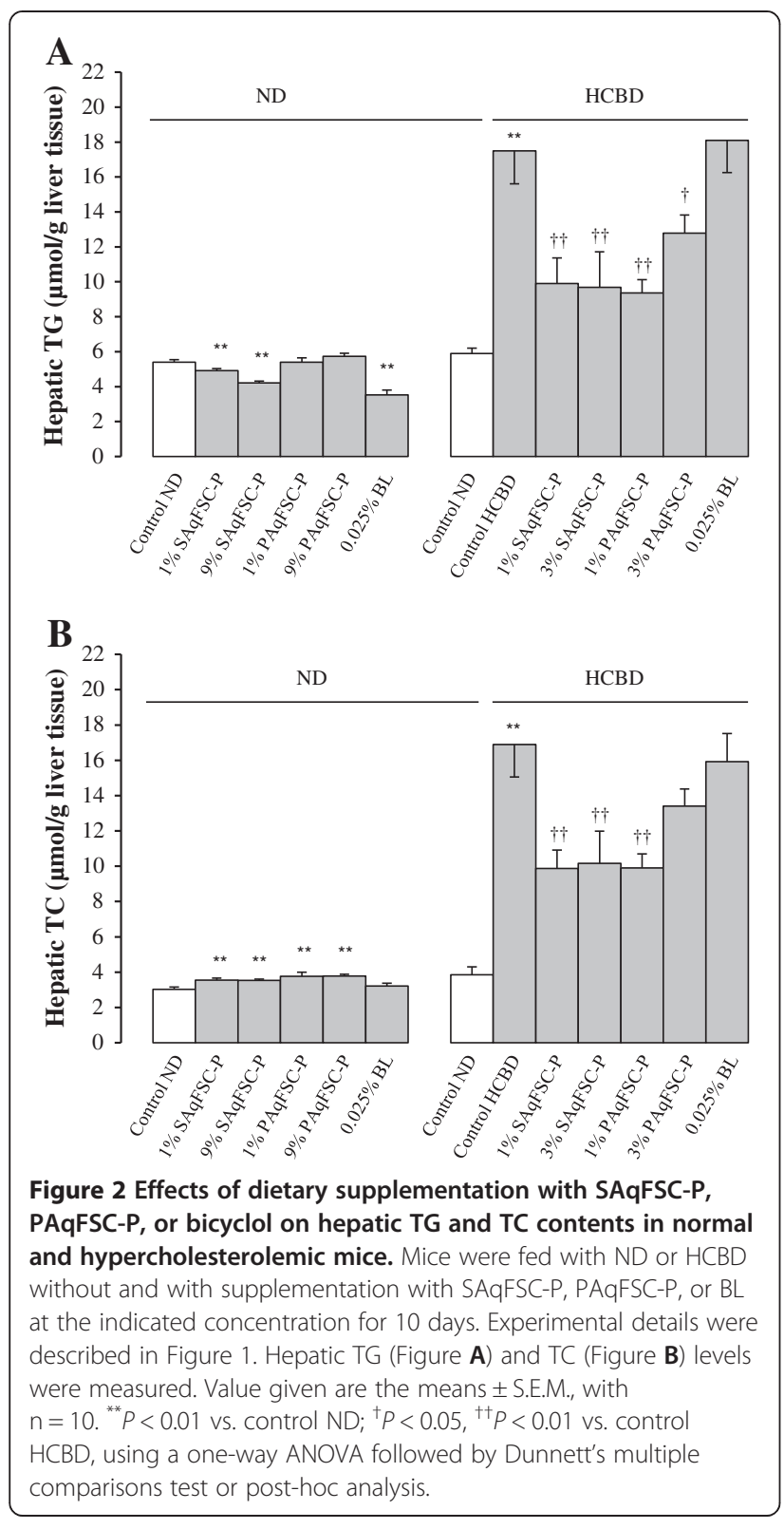

\section{Body weight gain and food/drug intake}

Supplementation with SAqFSC-P at 1\% or 9\% decreased the body weight gain during the period from D5 to D10 (by $23 \%$ and $21 \%$, respectively) in ND-fed mice. Mice fed with HCBD showed a significant decrease in body weight gain from D1 to D5 (by 6\%). The daily intake of SAqFSC$\mathrm{P}$ or PAqFSC-P was estimated to be $1.4-1.5 \mathrm{~g} / \mathrm{kg}$ (based on an equivalent amount of crude herb) at $1 \%$ supplementation, $4.5-4.7 \mathrm{~g} / \mathrm{kg}$ at $3 \%$ supplementation in HCBD-fed mice and $13.6-14.2 \mathrm{~g} / \mathrm{kg} /$ day at $9 \%$ supplementation in ND-fed mice. Bicyclol supplementation decreased the body weight gain from D5 to D10 (by 17\%) in ND-fed but not HCBD-fed mice. The daily intake of bicyclol was estimated to be $0.04 \mathrm{~g} / \mathrm{kg}$ (Table 3). SAqFSC-P and
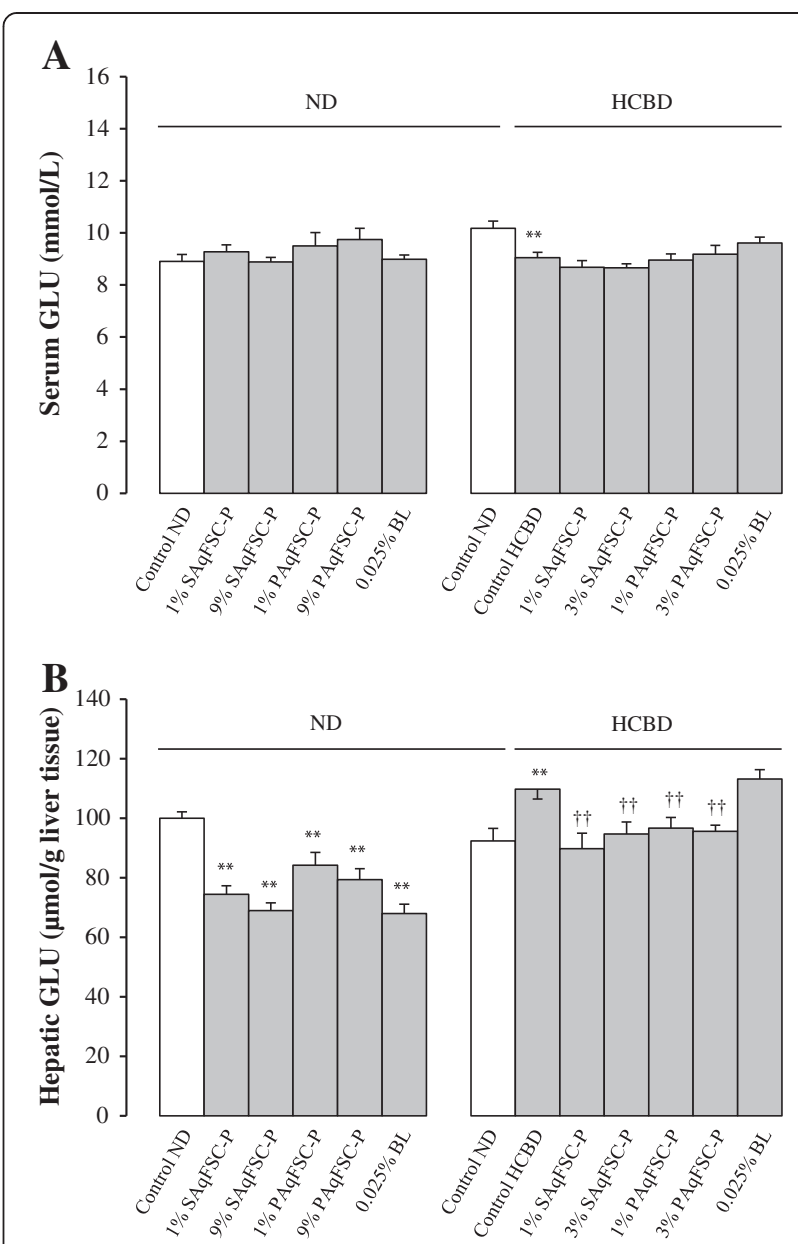

Figure 3 Effects of dietary supplementation with SAqFSC-P, PAqFSC-P, or bicyclol on serum and hepatic GLU contents in normal and hypercholesterolemic mice. Mice were fed with ND or HCBD without and with supplementation with SAqFSC-P or PAqFSC-P, or $\mathrm{BL}$ at the indicated concentration for 10 days. Experimental details were described in Figure 1. Serum (Figure A) and hepatic (Figure B) glucose (GLU) levels were measured. Value given are the means \pm S.E. M., with $n=10 .{ }^{* *} P<0.01$ vs. control ND; ${ }^{+\dagger} P<0.01$ vs. control HCBD, using a one-way ANOVA followed by Dunnett's multiple comparisons test or post-hoc analysis.

bicyclol supplementation did not reduce the body weight gain from D1 to D10.

\section{Discussion}

Hypercholesterolemia, which is a sub-type of hyperlipidemia characterized by significant elevation of serum TC level, is causally related to the pathogenesis of CHD, NAFLD, atherosclerosis, diabetes and metabolic syndrome $[20,21]$. Unhealthy eating habit is one major cause of hypercholesterolemia in humans. In this study, except for serum TG level, both serum and hepatic lipid levels were found to be increased by feeding HCBD in mice. Unlike other atherogenic diet containing cholesterol, sucrose, hydrogenated vegetable oil, sodium cholate, lactose, 
Table 2 Effects of dietary supplementation with SAqFSC-P, PAqFSC-P, or bicyclol on hepatic index, protein and function in normal and hypercholesterolemic mice

\begin{tabular}{lllll}
\hline Groups & Drug concentration $(\%, w / w)$ & Hepatic index (\%) & Hepatic protein (g/g liver tissue) & Serum ALT activity (U/L) \\
\hline Normal mice & & & & \\
Control ND & - & $6.59 \pm 0.10$ & $0.138 \pm 0.003$ & $36.50 \pm 3.61$ \\
SAqFSC-P/ND & 1 & $6.76 \pm 0.13$ & $0.131 \pm 0.003$ & $27.60 \pm 0.72^{\mathbf{a}}$ \\
& 9 & $6.91 \pm 0.13$ & $0.140 \pm 0.004$ & $30.33 \pm 2.41$ \\
PAqFSC-P/ND & 1 & $6.73 \pm 0.11$ & $0.146 \pm 0.002^{\mathbf{a}}$ & $32.80 \pm 1.63$ \\
& 9 & $7.16 \pm 0.17^{\mathbf{b}}$ & $0.155 \pm 0.003^{\mathbf{b}}$ & $32.00 \pm 1.81$ \\
Bicyclol/ND & 0.025 & $6.69 \pm 0.11$ & $0.153 \pm 0.002^{\mathbf{b}}$ & $26.00 \pm 1.67^{\mathbf{a}}$ \\
Hypercholesterolemic mice & & & $36.18 \pm 2.17$ \\
Control ND & - & $6.43 \pm 0.77$ & $0.133 \pm 0.004$ & $50.09 \pm 5.81^{\mathbf{a}}$ \\
Control HCBD & - & $7.19 \pm 0.13^{\mathbf{b}}$ & $0.150 \pm 0.002^{\mathbf{a}}$ & $47.26 \pm 3.58$ \\
SAqFSC-P/HCBD & 1 & $7.17 \pm 0.11$ & $0.140 \pm 0.005^{\mathbf{c}}$ & $46.32 \pm 2.52$ \\
PAqFSC-P/HCBD & 3 & $7.06 \pm 0.16$ & $0.153 \pm 0.003$ & $47.31 \pm 3.38$ \\
Bicyclol/HCBD & 1 & $6.99 \pm 0.16$ & $0.147 \pm 0.004$ & $43.60 \pm 3.90$ \\
\hline
\end{tabular}

Experimental details were described in Figure 1 and Table 1. Mice were fed with ND or HCBD containing SAqFSC-P, PAqFSC-P, or bicyclol at the indicated concentration for 10 days. Hepatic index (liver weight/body weight $\times 100$ ), protein (use Bradford assay) and function indicated by alanine aminotransferase (ALT) were then measured. Mouse model of hypercholesterolemia was generated by HCBD. Values given are the means \pm S.E.M., with $n=10$. ${ }^{\mathbf{a}} P<0.05$, ${ }^{\mathbf{b}} P<0.01$ vs. control ND; ${ }^{c} P<0.05$ vs. control HCBD, using a one-way ANOVA follow by Dunnett's multiple comparisons test or post-hoc analysis.

choline chloride, and thiouracil [22], which elevated serum TG and TC levels, HCBD used in this study did not increase serum TG levels. FSC-P (ie. SAqFSC-P and PAqFSC-P) intake for 10 days markedly reduced hepatic TC, TG, and GLU contents, as well as serum TG levels in

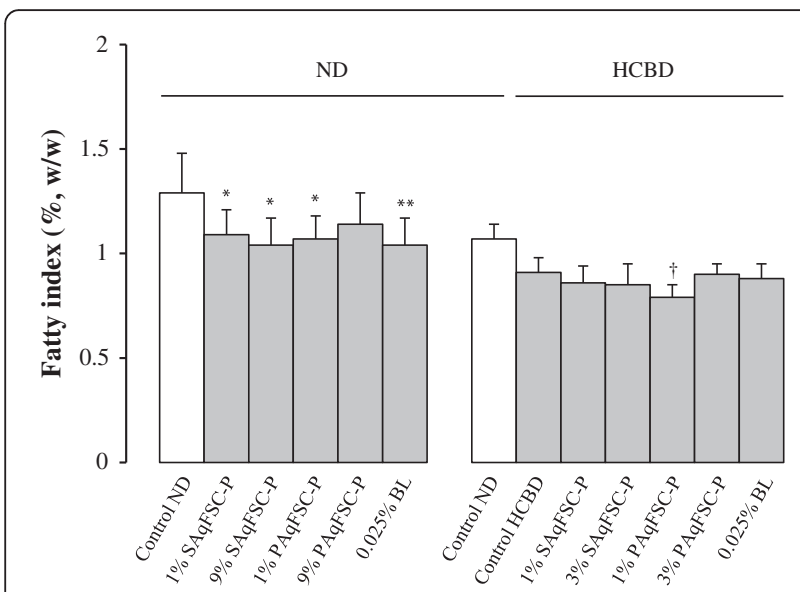

Figure 4 Effects of dietary supplementation with SAqFSC-P, PAqFSC-P, or bicyclol on fatty index in normal and hypercholesterolemic mice. Mice were fed with ND or HCBD without and with supplementation with SAqFSC-P, PAgFSC-P, or BL at the indicated concentration for 10 days. Experimental details were described in Figure 1. Fatty index was estimated from the ratio of epididymal fat weight to body weight (epididymal fat weight/body weight $\times 100$ ). value given are the means \pm S.E.M., with $n=10$. ${ }^{*} P<0.05,{ }^{* *} P<0.01$ vs. control ND; ${ }^{\dagger} P<0.05$ vs. control HCBD, using a one-way ANOVA followed by Dunnett's multiple comparisons test or post-hoc analysis. normal and hypercholesterolemic mice, as compared with the respective control mice, but it produced no significant effect on serum TC level. This observation suggested that the lipid-lowering effect of FSC-P may depend on basal lipid levels in the body. Our previous study has shown that schisandrin B, a major active ingredient presented in pulp fraction of FSC, lowered fat accumulation in L-02 cells incubated with free fat acid via inhibition of ADRP and SREBP-1 expression [23]. This might also explain the hepatic lipid-lowering effects of SAqFSC-P and PAqFSC-P supplementation. It has been demonstrated that FSC pulp mainly contains polysaccharides/sugars and organic acids, as well as dibenzocyclooctadiene lignans such as schisandrins (A, B, and C) and gomisins (A and N), which are believed to be the active ingredients in FSC [24,25]. In addition, several other dibenzocyclooctadiene lignans (Schisandra red pigment) have been isolated from the fruits of Schisandra wilsoniana [26,27].

LDL in the blood is originated from very low densityLDL (VLDL) produced in the liver in association with apoprotein B-100. Being associated with an increased risk of atherosclerosis and CHD, LDL is also called "bad cholesterol" [28,29]. In contrast, HDL, which is protective in nature, is called "good cholesterol". Hypercholesterolemia, including familial hypercholesterolemia, in human typically shows high levels of LDL and lower levels of HDL. In the current study, although the serum HDL level was not reduced, the LDL level was markedly elevated in hypercholesterolemic mice. Furthermore, LDL/HDL and 
Table 3 Effects of dietary supplementation with SAqFSC-P, PAqFSC-P, or bicyclol on body weight gain and food/drug intake in normal and hypercholesterolemic mice

\begin{tabular}{|c|c|c|c|c|c|}
\hline \multirow[t]{2}{*}{ Groups } & \multirow{2}{*}{$\begin{array}{l}\text { Drug concentration } \\
(\%, w / w)\end{array}$} & \multicolumn{2}{|c|}{ Body weight gain (g) } & \multirow{2}{*}{$\begin{array}{l}\text { Food intake } \\
\text { (g/kg/day) }\end{array}$} & \multirow{2}{*}{$\begin{array}{l}\text { Drug intak } \\
\text { (g/kg/day) }\end{array}$} \\
\hline & & D $1 \rightarrow$ D 5 & $\mathrm{D} 5 \rightarrow \mathrm{D} 10$ & & \\
\hline \multicolumn{6}{|l|}{ Normal mice } \\
\hline Control ND & - & $7.41 \pm 0.29$ & $4.46 \pm 0.22$ & 156.4 & - \\
\hline \multirow[t]{2}{*}{ SAqFSC-P/ND } & 1 & $7.52 \pm 0.07$ & $3.41 \pm 0.18^{\mathbf{a}}$ & 142.2 & 1.42 \\
\hline & 9 & $7.19 \pm 0.16$ & $3.53 \pm 0.27^{\mathbf{a}}$ & 157.5 & 14.18 \\
\hline \multirow[t]{2}{*}{ PAqFSC-P/ND } & 1 & $7.42 \pm 0.17$ & $3.99 \pm 0.36$ & 143.7 & 1.44 \\
\hline & 9 & $7.49 \pm 0.35$ & $4.54 \pm 0.26$ & 151.4 & 13.63 \\
\hline Bicyclol/ND & 0.025 & $7.41 \pm 0.20$ & $3.70 \pm 0.26^{\mathbf{a}}$ & 158.3 & 0.04 \\
\hline \multicolumn{6}{|c|}{ Hypercholesterolemic mice } \\
\hline Control ND & - & $10.42 \pm 0.25$ & $3.04 \pm 0.14$ & 162.7 & - \\
\hline Control HCBD & - & $9.76 \pm 0.16^{\mathbf{a}}$ & $2.81 \pm 0.12$ & 151.0 & - \\
\hline \multirow[t]{2}{*}{ SAqFSC-P/HCBD } & 1 & $9.71 \pm 0.29$ & $3.02 \pm 0.10$ & 151.4 & 1.51 \\
\hline & 3 & $9.36 \pm 0.20$ & $2.98 \pm 0.19$ & 150.0 & 4.50 \\
\hline \multirow[t]{2}{*}{ PAqFSC-P/HCBD } & 1 & $10.02 \pm 0.28$ & $2.78 \pm 0.11$ & 150.5 & 1.51 \\
\hline & 3 & $9.66 \pm 0.15$ & $3.11 \pm 0.16$ & 157.5 & 4.72 \\
\hline Bicyclol/HCBD & 0.025 & $10.36 \pm 0.23$ & $2.72 \pm 0.13$ & 152.5 & 0.04 \\
\hline
\end{tabular}

Experimental details were described in Figure 1. The dosages ( $\mathrm{g} / \mathrm{kg} /$ day, based on crude herbal material) were determined with respect to the amount of ingested diet (g/day $/ \mathrm{kg})$ and drug concentration in the diet. Values given are the means \pm S.E.M., with $n=10{ }^{a}{ }^{a}<0.05$ vs. control ND, using a one-way ANOVA followed by Dunnett's multiple comparisons test or post-hoc analysis.

$\mathrm{N}-\mathrm{HDL}$ values were increased, but HDL/LDL values were decreased in mice fed with HCBD-fed mice. As the predictive indicator of atherosclerosis and coronary artery calcium [30], high level of LDL and N-HDL caused by HCBD may hasten the development of hypercholesterolemia. In addition, the accumulation of fat in hepatic tissue may lead to liver damage in HCBD-fed mice, as indicated by an increase in serum ALT activity. FSC-P did not affect serum HDL and LDL levels in both normal and hypercholesterolemic mice, but PAqFSC-P decreased $\mathrm{N}$-HDL values in HCBD-fed mice. N-HDL cholesterol can predict the risk of CHD [31] and serves as a readily available parameter for estimating the risk of cognitive impairment in type 2 diabetic [32]. In this regard, PAqFSC$\mathrm{P}$ may be used for the prevention of hypercholesterolemia related CHD and memory disorders in patients with diabetes mellitus.

It has been well-known that lipid/fat metabolism has a close relationship with GLU metabolism. The relevant metabolic disorders constitute the pathological basis of modern epidemics such as hyperlipidemia, hyperglycemia, and hypertension, as well as metabolic syndrome, including obesity. In this study, HCBD-feeding was found to elevate serum TC and hepatic TC, TG, and GLU, as well as reduce serum GLU in mice. As such, the GLU metabolism is altered by hypercholesterolemic conditions. Studies have demonstrated that insulin resistance is the pathophysiological hallmark of NAFLD $[33,34]$. The suppression of insulin sensitivity results in the accumulation of GLU/ fat in the liver, and then leading to the development of NAFLD. As for HCBD-induced hypoglycemia may be an event secondary to the elevation in hepatic GLU contents. It is possible that release of hepatic GLU is inhibited in the HCBD-fed mice. Low hepatic GLU and lipid levels caused by FSC-P supplementation might result from the FSC-P-induced increase in hepatic insulin sensitivity in HCBD-fed mice [35,36]. FSC-P supplementation decreased the epididymal fat mass in mice fed with ND. However, due to the lowered fat index in HCBD-fed mice, FSC-P-induced fat loss was not observed in hypercholesterolemic mice. On the other hand, body weight loss was observed during D5 and D10 in ND-fed mice supplemented with SAqFSC-P/ ND. The mechanism underlying the FSC-P-induced loss in body weight/fat and hepatic lipid may also involve the inhibition of ADRP and SREBP-1 expression [23].

Previous studies have demonstrated that FSC related active compounds such as bicyclol, bifendate, and schisandrin $B$, when administered at high doses, can elevate serum TG level and hepatic weight in mice. As such, we have established new animal models of hyperlipidemia using schisandrin B [37-39] and bifendate [40]. However, FSC-P extracts used in the present study only produced minimal effect on serum TG level and hepatic weight. FSC-P at a high dose (9\%, up to $14 \mathrm{~g} / \mathrm{kg} /$ day for 10 days) did not affect animal behaviors (data not shown), which indicated the safety of FSC-P intake on central nervous system. In addition, serum creatinine levels, indicative of renal function, were not changed after FSC-P consumption 
(data not shown). Although we were not able to show a dose-response relationship in certain pharmacological activities of FSC-P, it was evident that FSC-P could significantly influence the hepatic lipid profile in hypercholesterolemic mice. Owing to the fact that chemical components in an herbal extract produce a wide spectrum of biological actions, the possible interactions among them may preclude the display of a dose-dependent relationship of a pharmacological activity. In the present study, some effects produced by SAqFSC-P or PAqFSC-P at high dose (3\% or $9 \%$ ) were less significant than at low dose $(1 \%)$. Therefore, caution has to be exercised in determining the dosage of FSC administration in clinical situations.
Despite the fact that biochemical mechanisms underlying the development of hyperlipidemia and its complications are widely investigated, hyperlipidemia remains the major cause of morbidity and mortality throughout the world. While the clinical management of hyperlipidemia has yet to be optimized, Chinese herbs, which have been used for thousands of years, may serve as an alternative treatment for hyperlipidemia. In the present study, the effect of FSC-P was investigated in the mouse model of hypercholesterolemia. Although both SAqFSC-P and PAqFSC-P supplementations notably lowered hepatic TG and GLU contents and elevated hepatic TC levels in ND-fed mice, no detectable changes were observed

Table 4 A summary of the current study

\begin{tabular}{|c|c|c|c|c|c|c|c|}
\hline \multirow[t]{2}{*}{ Groups } & \multicolumn{3}{|c|}{ Dietary SAqFSC-P supplement } & \multicolumn{3}{|c|}{ Dietary PAqFSC-P supplement } & \multirow{2}{*}{$\begin{array}{l}\text { Bicyclol } \\
0.025 \%\end{array}$} \\
\hline & $1 \%$ & $3 \%$ & $9 \%$ & $1 \%$ & $3 \%$ & $9 \%$ & \\
\hline \multicolumn{8}{|l|}{ Normal mice } \\
\hline Serum TC & - & & - & - & & - & - \\
\hline TG & - & & - & $\downarrow$ & & - & $\downarrow$ \\
\hline $\mathrm{HDL}$ & - & & - & - & & - & - \\
\hline LDL & - & & - & - & & - & - \\
\hline $\mathrm{N}-\mathrm{HDL}$ & - & & - & - & & - & - \\
\hline Glucose & - & & - & - & & - & - \\
\hline ALT activity & $\downarrow$ & & - & - & & - & $\downarrow$ \\
\hline Hepatic TC & $\uparrow$ & & $\uparrow$ & $\uparrow$ & & $\uparrow$ & - \\
\hline $\mathrm{TG}$ & $\downarrow$ & & $\downarrow$ & - & & - & $\downarrow$ \\
\hline Glucose & $\downarrow$ & & $\downarrow$ & $\downarrow$ & & $\downarrow$ & $\downarrow$ \\
\hline Index & - & & - & - & & $\uparrow$ & - \\
\hline Protein & - & & - & $\uparrow$ & & $\uparrow$ & $\uparrow$ \\
\hline Fat index & $\downarrow$ & & $\downarrow$ & $\downarrow$ & & - & $\downarrow$ \\
\hline Body weight gain & $\downarrow$ & & $\downarrow$ & - & & - & $\downarrow$ \\
\hline \multicolumn{8}{|c|}{ Hypercholesterolemic mice (change vs. normal) } \\
\hline Serum TC $(\uparrow)$ & - & $\downarrow$ & & - & - & & - \\
\hline $\mathrm{TG}(-)$ & $\downarrow$ & - & & $\downarrow$ & $\downarrow$ & & - \\
\hline $\mathrm{HDL}(-)$ & - & - & & - & - & & - \\
\hline $\operatorname{LDL}(\uparrow)$ & - & - & & - & - & & - \\
\hline $\mathrm{N}-\mathrm{HDL}(\uparrow)$ & - & - & & $\downarrow$ & $\downarrow$ & & - \\
\hline Glucose $(\downarrow)$ & - & - & & - & - & & - \\
\hline ALT activity $(\uparrow)$ & - & - & & - & - & & $\downarrow$ \\
\hline Hepatic TC $(\uparrow)$ & $\downarrow$ & $\downarrow$ & & $\downarrow$ & - & & - \\
\hline $\mathrm{TG}(\uparrow)$ & $\downarrow$ & $\downarrow$ & & $\downarrow$ & $\downarrow$ & & - \\
\hline Glucose $(\uparrow)$ & $\downarrow$ & $\downarrow$ & & $\downarrow$ & $\downarrow$ & & - \\
\hline Protein $(\uparrow)$ & $\downarrow$ & - & & - & - & & - \\
\hline Index $(\uparrow)$ & - & - & & - & - & & - \\
\hline Fat index $(-)$ & - & - & & $\downarrow$ & - & & - \\
\hline Body weight gain ( $\downarrow$ ) & - & - & & - & - & & - \\
\hline
\end{tabular}

TC: total cholesterol; TG: triglyceride; HDL: high-density lipoprotein; LDL: low-density lipoprotein; N-HDL: non-HDL; ALT: alanine aminotransferase. $\uparrow$ : increased or elevated; $\downarrow$ : decreased or inhibited; -: unaltered. 
in serum lipid and apolipoprotein levels. This finding suggests that the lipid-modulating effect of FSC-P may be influenced by factors such as lipid levels in tissues. However, it is still not clear whether dietary FSC-Pinduced increase in hepatic TC content is good or bad in ND-fed mice. Both SAqFSC-P and PAqFSC-P supplementations decreased serum TG levels and hepatic TC/TG contents in HCBD-fed mice, but no detectable effect on serum TC level was observed.

In conclusion, FSC pulp (i.e. SAqFSC-P or PAqFSC-P) supplementation ameliorated the fatty liver condition in HCBD-fed mice. FSC pulp supplementation decreased hepatic GLU and serum TG levels in both ND- and HCBD-fed mice. However, the supplementation with bicyclol did not affect serum and hepatic lipid levels in HCBD-fed mice (summarized in Table 4). SAqFSC-P and PAqFSC-P produce the similar beneficial outcomes in hypercholesterolemic mice. Naturally-occurring ingredients from FSC pulp may provide a safe alternative for the management of fatty liver and/or lipid disorders in humans.

\section{Materials and methods}

Herbal material and extraction procedure

FSC, the fruit of Schisandra Chinensis (Turcz.) Baill., was purchased from the Anguo Chinese herbs market and authenticated by professor Chun-Sheng Liu in the Beijing University of Chinese Medicine. The fruit pulp and seed were manually separated, then dried at room temperature. The weight of pulp and seed was $63 \%$ and $37 \%$ of total weight, respectively. FSC pulp (300 g) were powdered and soaked in $300 \mathrm{~mL}$ distilled water for $4 \mathrm{~h}$ at room temperature (approximately $25^{\circ} \mathrm{C}$ ), and they were then washed five times with $300 \mathrm{~mL}$ distilled water. The pooled aqueous extract was centrifuged at
$2000 \times g$ for $3 \mathrm{~min}$. The supernatant was concentrated under reduced pressure by rota-evaporation at $50^{\circ} \mathrm{C}$ to obtain $200 \mathrm{~mL}$ of SAqFSC-P (i.e., $0.95 \mathrm{~g}$ (dry weight) of FSC-P equivalent for every $1 \mathrm{~mL}$ of SAqFSC-P. As for the sediment, every $\mathrm{g}$ (wet weight) of PAqFSC-P was equivalent to $2.3 \mathrm{~g}$ of dried FSC-P. Both SAqFSC-P and PAqFSC-P were stored at $4^{\circ} \mathrm{C}$ until use (Figure 5).

\section{Chemicals and reagents}

Cholesterol (certificate no. 041103) and bile salt (certificate no. 000710) were obtained from Beijing Chemical Reagent Co. (Beijing, China). Fenofibrate (certificate no. 0405030) was bought from Beijing Yongkang Medical Ltd. (Beijing, China). Bicyclol was purchased from Beijing Union Pharm. (Beijing, China). Assay kits for TC (certificate no. 121471), TG (certificate no. 135991) and GLU (certificate no. 122991) were bought from Zhongsheng Beikong Bio-technology and Science Inc. (Beijing, China). Assay kits for HDL and LDL levels, as well as alanine aminotransferase (ALT) were obtained from Zhongsheng Beikong Bio-technology and Science Inc. (Beijing, China) or Beijing Leadman Biochemistry Co., Ltd. (Beijing, China). Coomassie brilliant blue G250 (certificate no. 20130624) was purchased from Nanjing Jiancheng Bioengineering Institute (Nanjing, China).

\section{Animal treatment}

Male ICR mice [Grade II, certificate No. SCXK (jing) 2006-0009], weighing 17-20 g (two-month-old), and the standard mouse chow diet were supplied by Vital River Lab Animal Co. Ltd. (Beijing, China). All animals were maintained on a $12 \mathrm{~h}$ (light on 700-1900 h) light-dark cycle at $20-21^{\circ} \mathrm{C}$, with a relative humidity of $50-55 \%$. They were allowed free access to water and food. Blood

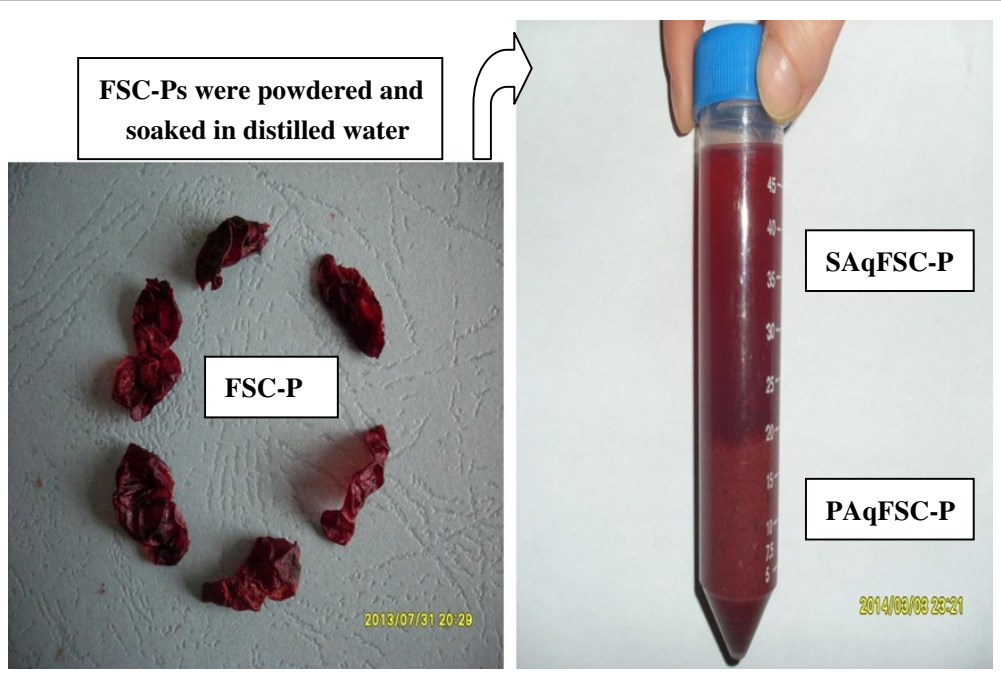

Figure 5 Preparation of SAqFSC-P and PAqFSC-P. FSC-P: Fructus Schisandrae Chinensis pulp; SAqFSC-P: supernatant of aqueous extract of FSC-P; PAqFSC-P: precipitate of aqueous extract of FSC-P. 
and liver/fat tissue samples were obtained from etheranesthetized animals which had been fasted for $6 \mathrm{~h}$ (from 600 to 1200). All experimental procedures were approved by the University Committee on Research Practice in Beijing University of Chinese Medicine.

\section{Study design 1: normal diet (ND)-fed mice}

This study was aimed to examine whether the oral administration of FSC-P could produce any toxic effects in mice fed with ND. Mice were divided into 6 groups of 10 animals in each: (1) control mice fed with ND; (2) and (3) mice fed with ND supplemented with $1 \%$ and 9\% (w/w) SAqFSC-P, respectively; (4) and (5) mice fed with ND supplemented with $1 \%$ and $9 \%$ PAqFSC-P, respectively; and (6) mice fed ND supplemented with 0.025\% bicyclol. Currently, bicyclol (a synthetic derivative of dibenzocyclooctadiene lignan from FSC) is used to treat hepatitis in China. Therefore, bicyclol was selected as a positive control. Mice were sacrificed ten days after the drug treatment.

\section{Study design 2: High cholesterol/bile salt diet (HCBD)-fed mice}

This study was aimed to investigate whether the oral administration of FSC-P could produce any beneficial effect on mice fed with HCBD containing cholesterol/ bile salt $(1 \% / 0.3 \%, w / w)$. Seventy mice were randomly assigned to seven groups of 10 animals in each: (1) control mice fed with ND; (2) control (ie. untreated) mice fed with HCBD; (3)-(6) mice fed with HCBD supplemented with SAqFSC-P or PAqFSC-P at $1 \%$ and $3 \%(w / w) ;(7)$ mice fed with HCBD supplemented with bicyclol at $0.025 \%$. Ten days later mice were killed and biochemical analyses were performed. Our previous studies showed that high levels of serum and hepatic lipids were observed in mice after 10 days on high-fat diet [15-19].

\section{Biochemical analysis}

Serum and liver samples were obtained $24 \mathrm{~h}$ after the last day of the experiment. Serum samples were prepared by centrifuging the whole blood obtained from the orbital vein for $8 \mathrm{~min}$ at $2000 \times g$ and stored at $-70^{\circ} \mathrm{C}$ until use for biochemical analyses. Liver tissue samples were homogenized in 9 volumes of saline solution by two 10 -s bursts of a tissue disintegrator at 13,500 rpm. The homogenates were then centrifuged at $2000 \times g$ for $15 \mathrm{~min}$ to obtain the supernatants. Thirty $\mu \mathrm{L}$ of the hepatic supernatant and $10 \mu \mathrm{L}$ serum was used to determine TG and TC levels with GPO-PAP and COD-PAP method, respectively. Ten $\mu \mathrm{L}$ serum and $5 \mu \mathrm{L}$ hepatic supernatant were used to determine the GLU levels with GOD-POD method. One hundred $\mu \mathrm{L}$ of hepatic supernatant were used to measure protein level using Bradford assay. Assays were performed by using assay kits according to the manufacturer's instructions. HDL/ LDL, LDL/HDL and N-HDL values were estimated by calculating the ratio of HDL to LDL and LDL to HDL, and the difference between TC and HDL, respectively. ALT activity was measured by automatic Biochemistry Analyzer (Beckman coulter Synchron CX4 PRO, Brea, CA, USA).

\section{Measurement of hepatic index and fatty index}

Liver weight was measured and hepatic index was estimated from the ratio of liver weight to body weight (liver weight/ body weight $\times 100$ ). Epididymal fat weight was measured and fatty index was estimated from the ratio of fat weight to body weight (epididymal fat weight/body weight $\times 100$ ).

\section{Statistical analysis}

Data were expressed as means \pm S.E.M., and they were analyzed by One-way ANOVA followed by Dunnett's multiple comparison test or post-hoc analysis using SPSS 16.0 statistical analysis program. $P<0.05$ was considered as significant.

\section{Abbreviations \\ NAFLD: Nonalcoholic fatty liver disease; FSC: Fructus Schisandrae Chinensis; SAqFSC-P: Supernatant of aqueous extract of FSC pulp; PAqFSC-P: Precipitate of aqueous extract of FSC pulp; TG: Triglyceride; TC: Total cholesterol; HDL: High-density lipoprotein cholesterol; LDL: Low-density lipoprotein cholesterol; GLU: Glucose; ALT: Alanine aminotransferase; CHD: Coronary heart disease.}

\section{Competing interests}

The authors declare that they have no competing interests.

\section{Authors' contributions}

Design of the study: SYP, ZLY; conduct of the study: NS, YZ, PLZ, XYW, ZSC; data collection: XYW, NS; data analysis: SYP, SFZ; data interpretation: SYP, KMK; manuscript writing: NS, SYP, KMK. All authors read and approved the final manuscript.

\section{Authors' information}

Si-Yuan Pan is a professor; Yi Zhang and Xiao-Yan Wang are Doctor Degree Graduate; Nan Sun, Zhu-Sheng Chu, and Pei-Li Zhu are Master Degree Graduate; Zhi-Ling Yu, PhD, is an associate professor; Shu-Feng Zhou, $\mathrm{PhD}$ and Kam-Ming Ko, PhD, are professors.

\section{Acknowledgements}

This work was supported by a grant from the National Natural Science Foundation of China (Grant No 31071989) and the Outstanding Teaching Team of Chinese Herbal Pharmacology in Beijing City (Grant No. 201006).

\section{Author details}

${ }^{1}$ Department of Pharmacology, School of Chinese Materia Medica, Beijing University of Chinese Medicine, Beijing 100102, China. ${ }^{2}$ School of Chinese Medicine, Hong Kong Baptist University, Hong Kong, China. ${ }^{3}$ Department of Pharmaceutical Sciences, College of Pharmacy, University of South Florida, Florida 33612, USA. ${ }^{4}$ Division of Life Science, Hong Kong University of Science \& Technology, Hong Kong, China.

Received: 14 November 2013 Accepted: 5 March 2014

Published: 12 March 2014

\section{References}

1. Nelson $\mathrm{RH}$ : Hyperlipidemia as a risk factor for cardiovascular disease. Prim Care 2013, 40(1):195-211.

2. Liu J, Zeng FF, Liu ZM, Zhang CX, Ling WH, Chen YM: Effects of blood triglycerides on cardiovascular and all-cause mortality: a systematic 
review and meta-analysis of 61 prospective studies. Lipids Health Dis 2013. doi:10.1186/1476-511X-12-159.

3. Vernon G, Baranova A, Younossi ZM: Systematic review: the epidemiology and natural history of non-alcoholic fatty liver disease and non-alcoholic steatohepatitis in adults. Aliment Pharmacol Ther 2011, 34(3):274-285.

4. Khoshdel AR, Carney SL, Gillies A: Circulatory syndrome: an evolution of the metabolic syndrome concept! Curr Cardiol Rev 2012, 8(1):68-76.

5. Pahan K: Biomedicine \& diseases: review lipid lowering drugs. Cell Mol Life Sci 2006, 63:1165-1178.

6. Amend KL, Landon J, Thyagarajan V, Niemcryk S, McAfee A: Incidence of hospitalized rhabdomyolysis with statin and fibrate use in an insured US population. Ann Pharmacother 2011, 45(10):1230-1239.

7. Enger C, Gately R, Ming EE, Niemcryk SJ, Williams L, McAfee AT: Pharmacoepidemiology safety study of fibrate and statin concomitant therapy. Am J Cardiol 2010, 106(11):1594-1601.

8. Potgieter M, Pretorius $E_{1}$ Pepper MS: Primary and secondary coenzyme Q10 deficiency: the role of therapeutic supplementation. Nutr Rev 2013, 71(3):180-188.

9. Scholle JM, Baker WL, Talati R, Coleman Cl: The effect of adding plant sterols or stanols to statin therapy in hypercholesterolemic patients: systematic review and meta-analysis. J Am Coll Nutr 2009, 28(5):517-524

10. Saez Lancellotti TE, Boarelli PV, Romero AA, Funes AK, Cid-Barria M, Cabrillana ME, Monclus MA, Simón L, Vicenti AE, Fornés MW: Semen quality and sperm function loss by hypercholesterolemic diet was recovered by addition of olive oil to diet in rabbit. PLoS One 2013, 8(1):e52386.

11. Schmidt S, Willers J, Stahl F, Mutz KO, Scheper T, Hahn A, Schuchardt JP: Regulation of lipid metabolism-related gene expression in whole blood cells of normo- and dyslipidemic men after fish oil supplementation. Lipids Health Dis 2012. doi:10.1186/1476-511X-11-172.

12. Awaisheh SS, Khalifeh MS, Al-Ruwaili MA, Khalil OM, Al-Ameri OH, Al-Groom R Effect of supplementation of probiotics and phytosterols alone or in combination on serum and hepatic lipid profiles and thyroid hormones of hypercholesterolemic rats. J Dairy Sci 2013, 96(1):9-15

13. Hasani-Ranjbar S, Nayebi N, Moradi L, Mehri A, Larijani B, Abdollahi M: The efficacy and safety of herbal medicines used in the treatment of hyperlipidemia: a systematic review. Curr Pharm Des 2010, 16(26):2935-2940.

14. Singh BB, Vinjamury SP, Der-Martirosian C, Kubik E, Mishra LC, Shepard NP, Singh VJ, Meier M, Madhu SG: Ayurvedic and collateral herbal treatments for hyperlipidemia: a systematic review of randomized controlled trials and quasi-experimental designs. Altern Ther Health Med 2007, 13(4):22-28.

15. Pan SY, Yu Q, Zhang Y, Wang XY, Sun N, Yu ZL, Ko KM: Dietary fructus Schisandrae extracts and fenofibrate regulate the serum/hepatic lipid-profile in normal and hypercholesterolemic mice, with attention to hepatotoxicity. Lipids Health Dis 2012. doi:10.1186/1476-511X-11-120.

16. Pan SY, Yu ZL, Dong H, Xiang CJ, Fong WF, Ko KM: Ethanol extract of Fructus Schisandrae decreases hepatic triglyceride level in mice fed with a high fat/cholesterol diet, with attention to acute toxicity. Evid Based Complement Alternat Med 2011. doi:10.1093/ecam/nep070.

17. Pan SY, Dong $H$, Zhao XY, Xiang CJ, Fang HY, Fong WF, Yu ZL, Ko KM: Schisandrin $B$ from Schisandra chinensis reduce hepatic lipid contents in hypercholesterolaemic mice. J Pharm Pharmacol 2008, 60(3):399-403.

18. Pan SY, Dong H, Yu ZL, Zhao XY, Xiang CJ, Wang H, Fong WF, Ko KM: Bicyclol, a synthetic dibenzocyclooctadiene derivative, decreases hepatic lipids but increases serum triglyceride level in normal and hypercholesterolaemic mice. J Pharm Pharmacol 2007, 59(12):1657-1662.

19. Pan SY, Yang R, Dong H, Yu ZL, Ko KM: Bifendate treatment attenuates hepatic steatosis in cholesterol/bile salt- and high-fat diet-induced hypercholesterolemia in mice. Eur J Pharmacol 2006, 552(1-3):170-177.

20. Robinson JG: Management of familial hypercholesterolemia: a review of the recommendations from the National Lipid Association Expert Panel on Familial Hypercholesterolemia. J Manag Care Pharm 2013, 19(2):139-149.

21. Wang J, Chiu WH, Chen RC, Chen FL, Tung TH: The clinical investigation of disparity of nonalcoholic fatty liver disease in a Chinese occupational population in Taipei, Taiwan: experience at a teaching hospital. Asia Pac J Public Health 2013. doi:10.1177/1010539513483830.

22. da Motta NA V, Kümmerle AE, Marostica E, Fernandes Dos Santos C, Manssour Fraga CA, Barreiro EJ, de Miranda AL, de Brito FC: Anti-atherogenic effects of a new thienylacylhydrazone derivative, LASSBio-788, in rats fed a hypercholesterolemic diet. J Pharmaco/ Sci 2013 [Epub ahead of print].
23. Chu JH, Wang H, Ye Y, Chan PK, Pan SY, Fong WF, Yu ZL: Inhibitory effect of schisandrin B on free fatty acid-induced steatosis in L-02 cells. World J Gastroenterol 2011, 17(19):2379-2388.

24. Li LS, Shi WJ, Ma XB: Comparative analysis of bioactive component in seeds and flesh of Schisandrae chinensis Fructus by thin layer chromatography. Food Sci 2012, 33(6):175-178.

25. Li L, Xiao Y, Yu D, Ma Y, Huang W, Tian G, Chen L: Determination of three organic acids in Schisandrae Chinensis Fructus by HPLC. Chin J Chin Materia Med 2011, 36(23):3286-3289.

26. Yang GY, Li YK, Wang RR, Xiao WL, Yang LM, Pu JX, Zheng YT, Sun HD: Dibenzocyclooctadiene lignans from the fruits of Schisandra wilsoniana and their anti-HIV-1 activities. J Asian Nat Prod Res 2010, 12(6):470-476.

27. Yang GY, Wang RR, Mu HX, Li YK, Li XN, Yang LM, Zheng YT, Xiao WL, Sun HD: Dibenzocyclooctadiene lignans and norlignans from fruits of Schisandra wilsoniana. J Nat Prod 2013, 76(2):250-255.

28. Rizzo M, Berneis K: Low-density lipoprotein size and cardiovascular risk assessment. QJM 2006, 99(1):1-14.

29. Martin SS, Blaha MJ, Elshazly MB, Brinton EA, Toth PP, McEvoy JW, Joshi PH, Kulkarni KR, Mize PD, Kwiterovich PO, Defilippis AP, Blumenthal RS, Jones SR: Friedewald-estimated versus directly measured low-density. J Am Coll Cardiol 2013, 62(8):732-739.

30. Simprini $L A$, Villines $T C$, Rich $M$, Taylor AJ: The relationship between subclinical atherosclerosis, non-high-density lipoprotein cholesterol, exercise, and diet among male participants of the PACC Project. J Clin Lipidol 2012, 6:174-179

31. Sondermeijer BM, Rana JS, Arsenault BJ, Shah PK, Kastelein JJ, Wareham NJ, Boekholdt SM, Khaw KT: Non-HDL cholesterol vs. Apo B for risk of coronary heart disease in healthy individuals: the EPIC-Norfolk prospective population study. Eur J Clin Invest 2013. doi: 10.1111/eci.12129.

32. Niu MJ, Yin FZ, Liu LX, Fang Y, Xuan XM, Wu GF: Non-high-density lipoprotein cholesterol and other risk factors of mild cognitive impairment among Chinese type 2 diabetic patients. J Diabetes Complications 2013. doi:10.1016/j.jdiacomp.

33. Bugianesi E, Moscatiello S, Ciaravella MF, Marchesini G: Insulin resistance in nonalcoholic fatty liver disease. Curr Pharm Des 2010, 16(17):1941-1951.

34. Gaggini M, Morelli M, Buzzigoli E, DeFronzo RA, Bugianesi E, Gastaldelli A Non-alcoholic fatty liver disease (NAFLD) and its connection with insulin resistance, dyslipidemia, atherosclerosisand coronary heart disease. Nutrients 2013, 5(5):1544-1560.

35. Kwon DY, da Kim S, Yang HJ, Park S: The lignan-rich fractions of Fructus Schisandrae improve insulin sensitivity via the PPAR $-\gamma$ pathways in in vitro and in vivo studies. J Ethnopharmacol 2011, 135(2):455-462.

36. Park S, Hong SM, Ahn IS, Kim YJ, Lee JB: Huang-Lian-Jie-Du-Tang supplemented with Schisandra chinensis Baill. and Polygonatum odoratum Druce improved glucose tolerance by potentiating insulinotropic actions in islets in 90\% pancreatectomized diabetic rats. Biosci Biotechnol Biochem 2009, 73(11):2384-2392.

37. Pan SY, Dong H, Han YF, Li WY, Zhao XY, Ko KM: A novel experimental model of acute hypertriglyceridemia induced by schisandrin B. Eur J Pharmacol 2006, 537(1-3):200-2044.

38. Pan SY, Jia ZH, Zhang Y, Yu Q, Wang XY, Sun N, Zhu PL, Yu ZL, Ko KM: A novel mouse model of combined hyperlipidemia associated with steatosis and liver injury by a single-dose intragastric administration of schisandrin B/cholesterol/bile salts mixture. J Pharmacol Sci 2013, 123(2):110-119.

39. Pan SY, Dong $H$, Guo BF, Zhang Y, Yu ZL, Fong WF, Han YF, Ko KM: Effective kinetics of schisandrin $B$ on serum/hepatic triglyceride and total cholesterol levels in mice with and without the influence of fenofibrate. Naunyn Schmiedebergs Arch Pharmacol 2011, 383(6):585-591.

40. Pan SY, Yang R, Han YF, Dong H, Feng XD, Li N, Geng W, Ko KM: High doses of bifendate elevate serum and hepatic triglyceride levels in rabbits and mice: animal models of acute hypertriglyceridemia. Acta Pharmacol sin 2006, 27(6):673-678.

\section{doi:10.1186/1476-511X-13-46}

Cite this article as: Sun et al.: Dietary pulp from Fructus Schisandra Chinensis supplementation reduces serum/hepatic lipid and hepatic glucose levels in mice fed a normal or high cholesterol/bile salt diet. Lipids in Health and Disease 2014 13:46. 